Alma Mater Studiorum - Università di Bologna DEPARTMENT OF ECONOMICS

\title{
When perfectionism becomes willpower
}

Daniele Pennesi

Quaderni - Working Paper DSE $N^{\circ} 1050$

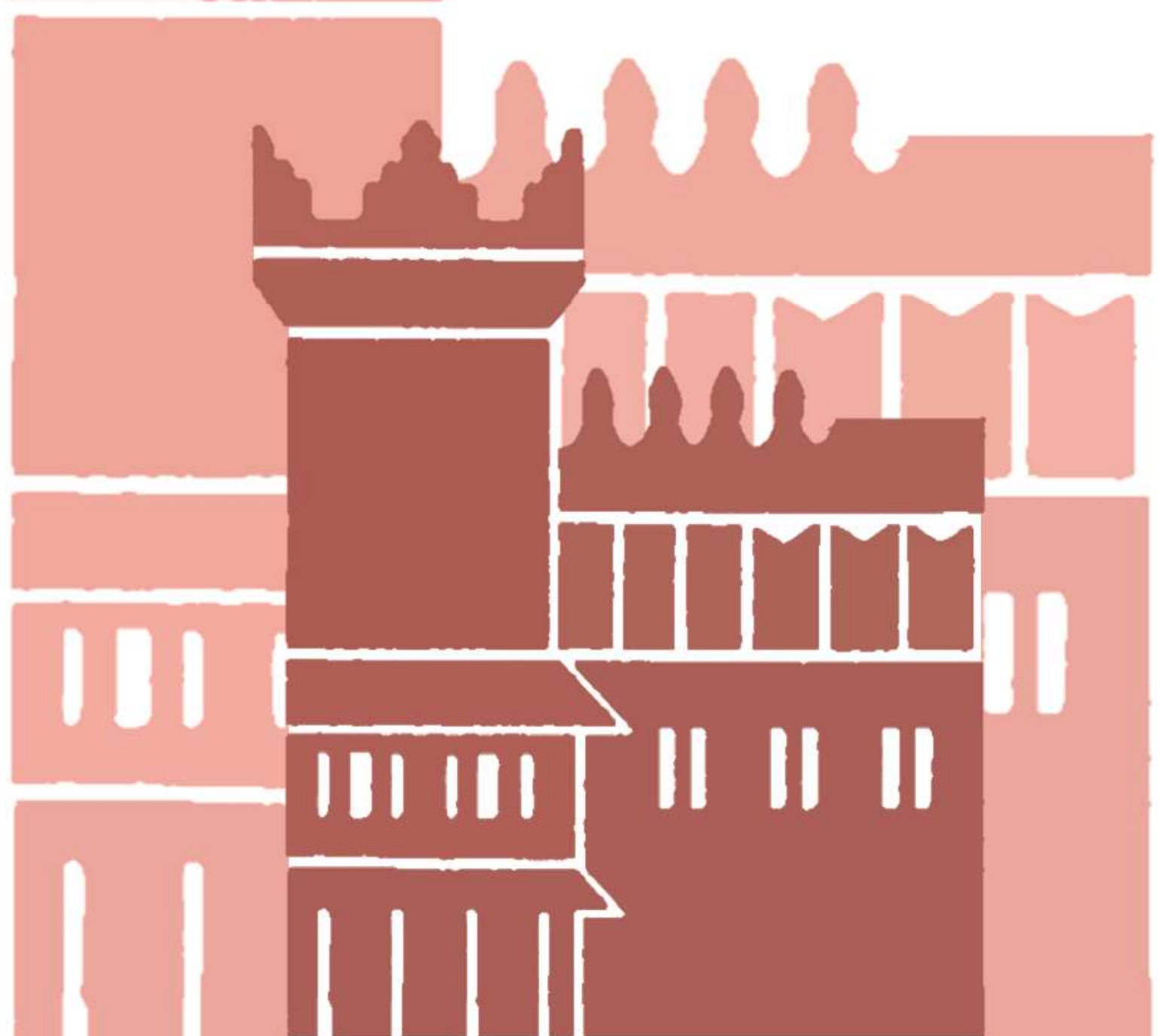




\title{
When perfectionism becomes willpower*
}

\author{
Daniele Pennesi ${ }^{\dagger}$
}

January 2016

\begin{abstract}
Perfectionism can be healthy: striving for perfection requires the ability to selfregulate, namely willpower. This paper formalizes the intuitive relation between healthy perfectionism and willpower in the presence of temptation. The value of a menu of options for an individual with limited willpower corresponds to the lower bound of the value assigned to the same menu by a perfectionist, when temptation and perfectionism intensities are free to vary. Moreover, the higher the perfectionism strive, the higher the willpower. The relation between overwhelming temptation and the Strotz model is a particular case of the result. When there is uncertainty about temptation, we generalize Dekel and Lipman (2012) providing conditions such that a preference is represented by a random willpower representation, if and only if, it has an equivalent random perfectionism representation.
\end{abstract}

KEYWORDS: Temptation, perfectionism, limited willpower Jel Classification: D01

${ }^{*}$ I thank Igor Kopylov and Todd Sarver for helpful suggestions.

${ }^{\dagger}$ University of Bologna, Department of Economics, Piazza Scaravilli 2, 40126, Bologna (Italy). Email: daniele.pennesi@unibo.it 


\section{Perfectionism and willpower}

Perfectionism refers to a psychological trait characterized by high personal standards, often coupled with an overly critical evaluation of own performance (Frost et al., 1990). In the economic literature studying temptation, perfectionism has been proposed as an explanation for a behavior that is often attributed to naivety. A perfectionist derives utility from the inclusion of a normatively optimal option, although it won't be selected later (Kopylov, 2012). Similarly to naivety, this implies a desire for flexibility that is not used in the future (see DellaVigna and Malmendier (2006), for an example). Willpower refers to a stock of mental resources that are used to resist temptation (Baumeister et al., 1998). Exerting self-control is costly and individuals give in to temptations when the cost of resisting is higher than their stock of willpower. When evaluating a set of options, the individual focuses on the subset containing those elements whose "cost of temptation" is smaller than her stock of willpower.

This paper shows an unexpected relation between models of choice among menus accounting for limited willpower and models of perfectionist choice. The value of a menu according to the willpower model is the lower bound for the value of the menu according to the perfectionist model, when temptation and perfectionism intensities are free to vary. In addition, the stock of willpower is equal to the "degree of perfectionism". In this sense, perfectionism becomes willpower. In the case of no perfectionism, the result corresponds to the relation between the temptation model of Gul and Pesendorfer (2001), henceforth GP, and the Strotz model. They prove that the Strotz model arises from GP when the temptation's intensity becomes overwhelming. According to our approach, the Strotz value of a menu is the lower bound of the value assigned to the menu by the GP model, when the temptation's intensity varies. The previous limiting result is robust to the introduction of stochastic temptation. Moreover, we generalize the result of Dekel and Lipman (2012), showing that a preference has a sufficiently smooth random willpower representation, if and only if, it has an equivalent capped random perfectionism representation (see Theorem 4). Hence, we provide a deeper relation between models of perfectionism and limited willpower with stochastic temptation. A final application to the dynamic of willpower shows how the effect of exerting self-control today can influence the stock of willpower tomorrow. If 
willpower depletes with use, a steady-state of (random) self-indulgence arises, although temptations' intensity is constant across time. If willpower is "trained" with use, as suggested in Baumeister et al. (1998), preferences in the steady-state are equivalent to a pure preference for flexibility.

The paper makes several contributions. The first is technical, since we connect a well-established literature on subdifferential and $\epsilon$-subdifferential calculus (Zalinescu, 2002) to models of choice among menus. The machinery developed in this work and the techniques developed in subdifferential calculus can be readily applied to other models of choice among menus, for example, those relaxing the independence axiom (Ergin and Sarver, 2010; Noor and Takeoka, 2015) and in different areas, such as the menu-approach to ambiguity (Ahn, 2008; Gajdos et al., 2008) and rational inattention (De Oliveira et al., 2013; Pennesi, 2015).

Secondly, the link we establish between the two classes of models, one studying perfectionism and the other willpower, is not a mere technical result. It is motivated by the psychological literature viewing perfectionism as an "adaptive" trait, also called positive striving (Hamachek, 1978; Stoeber and Otto, 2006). Positive striving forces individuals to set and pursue high standards, this requires a high capacity of self-regulation. In turn, the ability to self-regulate is directly related to willpower, since more willpower generates a higher ability to self-regulate. Therefore, the paper proposes a formalization of a link that can be found informally in the psychological literature. The paper also contributes to the growing literature studying models of choice with limited willpower (Masatlioglu et al., 2011; Ozdenoren et al., 2012). Since the axiomatic foundation of perfectionism is known (see Kopylov, 2012), the relation we propose in this work may inform future attempts to lay down the axiomatic foundations of models of limited willpower. Lastly, the model of willpower we introduce here has alternative interpretations: it can be thought as the first-period choice of an individual who anticipates choice overload in the second-period (see Frick, 2015), or anticipates a "satisficing" rule $^{1}$ (Aleskerov et al., 2007; Manzini et al., 2013). Therefore, the results of the paper have a wide range of alternative applications and they

\footnotetext{
${ }^{1}$ In the satisficing rule of Aleskerov et al. (2007), an individual chooses form a set $x$ according to $C(x)=$ $\{p \in x: v(p) \geq \delta(x)\}$. If $\delta(x)=\max _{q \in x} v(q)-w(x)$, we recover the willpower representation. In the twostage threshold representation of Manzini et al. (2013), the choice rule is $C(x)=\operatorname{argmax}_{p \in x} g(p)$ such that $f(p) \geq \theta(x)$.
} 
are useful to study models of choice departing from the "temptation" approach.

\section{$1.1 \quad$ Related literature}

Gul and Pesendorfer (2001) introduced temptation in a model of choice among menus. Resisting temptation has a menu-dependent cost and an individual may prefer smaller to larger menus. Their model includes as a particular case the Strotz's model: when the temptation's intensity becomes overwhelming, the Gul and Pesendorfer (2001) model becomes the Strotz model. We show that this result is a particular case of a more general relation linking perfectionism and willpower. Perfectionism has been introduced in Kopylov (2012) to account for the empirical evidence showing a desire for flexibility in the presence of temptation, for example, buying an expensive monthly pass for a gym, whereas daily passes would be more convenient given the actual number of visits (DellaVigna and Malmendier, 2006). Perfectionism offers an explanation for the latter behavior that does not rely on naivety. ${ }^{2}$ A perfectionist benefits from including normatively optimal options, although those are not selected in the secondperiod choice. Dekel and Lipman (2012) introduced a stochastic version of the Strotz model, where uncertainty affects future temptations. They establish a surprising relation between the random Strotz and the random GP representations (a generalization of the GP model with uncertainty about temptation). A preference has a sufficiently smooth random Strotz representation, if and only if, it has an equivalent random GP representation. We generalize this equivalence providing conditions such that a preference has a sufficiently smooth random Strotz with willpower representation, if and only if, it has a random temptation with perfectionism representation. Concerning the theoretical literature on willpower, Ozdenoren et al. (2012) modeled dynamic choices in continuous time where willpower acts as a stock of resources devoted to resist temptation. Two models of choice from menus, Masatlioglu et al. (2011) and Frick (2015), the latter with a different interpretation, can be interpreted as the choice rules induced in the second-period by willpower models. In the case of Masatlioglu et al. (2011) with a menu-independent stock of willpower ${ }^{3}$ and, in the case of Frick (2015), with a menu-

\footnotetext{
${ }^{2} \mathrm{~A}$ naive individual overestimates his ability to resist temptation.

${ }^{3}$ In an early version of their paper, Masatlioglu et al. (2011) axiomatized a model of choice among menus with limited and menu-independent willpower.
} 
dependent stock. An axiomatization of a model of choice among menus that accounts for limited willpower is still missing in the literature. This paper contributes to this goal providing a relation between limited willpower and perfectionism.

\section{Temptation, perfectionism and willpower}

Let $Z$ be a finite set of prizes, a lottery is an element $p$ of the simplex $\Delta(Z)$. A menu $x$ is a compact, convex and nonempty subset of $\Delta(Z)$. The set of all menus is denoted by $\mathcal{X}$. A preference relation $\succcurlyeq$ is defined over $\mathcal{X}$. A representation of a preference $\succcurlyeq$ is a function $V: \mathcal{X} \rightarrow \mathbb{R}$, such that $V(x) \geq V(y)$, if and only if, $x \succcurlyeq y$.

We begin introducing the Perfectionism representation $V_{P}$ of Kopylov (2012). It evaluates a menu $x \in \mathcal{X}$ according to:

$$
V_{P}(x)=\max _{p \in x}[u(p)+v(p)]-\max _{q \in x} v(q)+k \max _{q \in x} u(q)
$$

where $u, v: \Delta(Z) \rightarrow \mathbb{R}$ are expected utility functions and $k>-1$. The function $u$ is interpreted as the normative utility and $v$ as the temptation ranking $(v(p) \geq v(q)$, if and only if, $p$ is more tempting than $q$ ). The parameter $k$ models the effect of perfectionism, if $k>0$, the individual has a preference for flexibility, i.e. $x \cup y \succ x \succcurlyeq$ $y$, for some $x, y \in \mathcal{X}$, (see Kopylov, 2012), even though such flexibility will not be used in the future. ${ }^{4}$ A Gul-Pesendorfer (GP) representation $V_{G P}$ is a Perfectionism representation with $k=0$ :

$$
V_{G P}(x)=\max _{p \in x}[u(p)+v(p)]-\max _{q \in x} v(q)
$$

A Willpower representation evaluates a menu according to:

$$
V_{W}(x)=\max _{p \in B(x ; v, w(x))} u(p)
$$

where

$$
B(x ; v, w(x))=\left\{p \in x: w(x) \geq \max _{q \in x} v(q)-v(p)\right\}
$$

\footnotetext{
${ }^{4}$ Given sophistication (and other axioms), the second-period choice function associated with $V_{P}$ is $C(x)=\operatorname{argmax}_{p \in x} u(p)+v(p)$, and it is identical to the choice rule associated with a GP representation. Perfectionism affects the ex-ante value of a menu, but it does not affect the second-period choice.
} 
$w(x) \geq 0$ for all $x \in \mathcal{X}$. The value of a menu is equal to the maximum normative utility $u$ achievable on a restricted menu containing the elements whose cost, in terms of resisting temptation, $\max _{q \in x} v(q)-v(p)$, is smaller than the stock of willpower $w(x)$. If $w(x)=w \geq 0$ for all $x \in \mathcal{X}$, we call the previous model the Limited Willpower model. If $w(x)=w=0$ for all $x \in \mathcal{X}$, the Willpower model becomes the Strotz model (Strotz, 1956),

$$
V_{S}(x)=\max _{p \in B(x ; v, 0)} u(p)
$$

where $B(x ; v, 0)=\left\{p \in x: v(p) \geq \max _{q \in x} v(q)\right\}$. The model of willpower acts as a bridge between a pure preference for flexibility in the sense of Kreps (1979) and the overwhelming temptation of Strotz. Indeed, for large enough $w(x), B(x ; v, w(x))=x$, hence $V_{W}(x)=\max _{p \in x} u(p)$ as in Kreps, whereas for $w(x)=0, V_{W}(x)=V_{S}(x)$. As anticipated in the introduction, the willpower model can be alternatively interpreted as a first-period choice of an individual who anticipates choice overload (Frick, 2015), or anticipates a "satisficing" rule (Aleskerov et al., 2007), or anticipates a two-stage with threshold choice rule (Manzini et al., 2013).

It is well known (Gul and Pesendorfer, 2001) that the Strotz representation is a limiting case of the GP representation:

$$
\lim _{\lambda \rightarrow \infty}\left[\max _{p \in x}[u(p)+\lambda v(p)]-\lambda \max _{q \in x} v(q)\right]=\max _{p \in B(x ; v, 0)} u(p)
$$

When the intensity of the temptation becomes overwhelming, the individual anticipates her inability to resist and she only considers the restricted menu containing elements that maximize the temptation utility. The main result of the paper follows from the observation that the relation between the GP and the Strotz representation can be rewritten as (see Eq. (2)):

$$
\inf _{\lambda>0}\left[\max _{p \in x}\left[u(p)+\frac{1}{\lambda} v(p)\right]-\frac{1}{\lambda} \max _{q \in x} v(q)\right]=\max _{p \in B(x ; v, 0)} u(p)
$$

The interpretation of the previous expression is the following: the Strotz's evaluation of a menu $x \in \mathcal{X}$ is equal to the lower bound of the evaluation of the same menu according to GP, when the intensity of the temptation is free to vary. In other words, the Strotz value of a menu corresponds to the worst possible value assigned by a GP 
to a menu, when the temptation intensity changes. Using the previous consideration, we can relate the perfectionism and the willpower models.

As for the GP case, one can be interested in the lower bound for the value of a menu, when the intensity of the temptation and the intensity of perfectionism vary. Formally, for a given $x \in \mathcal{X}$, consider the expression:

$$
\inf _{\lambda>0}\left[\max _{p \in x}\left[u(p)+\frac{1}{\lambda} v(p)\right]-\frac{1}{\lambda} \max _{q \in x} v(q)+\frac{1}{\lambda} k \max _{q \in x} u(q)\right]
$$

The following result shows that this value is equal to the value assigned by the willpower representation to $x$, with a particular stock of willpower. ${ }^{5}$

Theorem 1. For all $x \in \mathcal{X}$, if $k \max _{q \in x} u(q) \geq 0$,

$$
\left.\inf _{\lambda>0}\left[\max _{p \in x}\left[u(p)+\frac{1}{\lambda} v(p)\right]-\frac{1}{\lambda} \max _{q \in x} v(q)\right]+\frac{1}{\lambda} k \max _{q \in x} u(q)\right]=\max _{p \in B(x ; v, w(x))} u(p)
$$

where $w(x)=k \max _{q \in x} u(q)$.

It follows that the lowest value of a menu $x$ for a perfectionist corresponds to the value given by the willpower model, where the stock of willpower is the "value of perfectionism" $k \max _{q \in x} u(q)$. In this sense: perfectionism becomes willpower. Suppose $\max _{q \in x} u(q) \geq 0$, since, $B(x ; v, w(x))=\left\{p \in x: k \max _{q \in x} u(q) \geq \max _{q \in x} v(q)-v(p)\right\}$, the larger the craving for perfectionism, parametrized by $k$, the larger is the stock of willpower. As a limiting case, for a large enough value of perfectionism, for example, $k \max _{q \in x} u(q) \geq \max _{p \in x}\left(\max _{q \in x} v(q)-v(p)\right)$, we have $B(x ; v, w(x))=x$ and the value of $x$ is equal to that assigned by a pure preference for flexibility representation. The previous result provides information about the axiomatic foundation of the willpower model. Being a particular case of the perfectionism representation, it has to satisfy the Independence and the Perfection Set-Betweenness axiom ${ }^{6}$ of Kopylov (2012). A particular case of Theorem 1 offers a new version of the relation between Strotz and the GP model.

\footnotetext{
${ }^{5}$ The proof follows directly from Theorem 2 .

${ }^{6}$ For all $p \in \Delta(Z)$ and $x, y \in \mathcal{M}_{p}, x \succcurlyeq y$ implies $\{p\} \succcurlyeq x \succcurlyeq x \cup y \succcurlyeq y$. Where $\mathcal{M}_{p}=$ $\{x \in \mathcal{X}: p \in x,\{p\} \succcurlyeq\{q\}, \forall q \in x\}$.
} 
Corollary 1. If $k=0$, i.e. no-perfectionism,

$$
\left.\inf _{\lambda>0}\left[\max _{p \in x}\left[u(p)+\frac{1}{\lambda} v(p)\right]-\frac{1}{\lambda} \max _{q \in x} v(q)\right]\right]=\max _{p \in B(x ; v, 0)} u(p)
$$

where $B(x ; v, 0)=\left\{p \in x: v(p) \geq \max _{q \in x} v(q)\right\}$.

The link with the overwhelming approach of GP follows from Eq. (2). Indeed,

$$
\begin{aligned}
\left.\lim _{\hat{\lambda} \rightarrow \infty}\left[\max _{p \in x}[u(p)+\hat{\lambda} v(p)]-\hat{\lambda} \max _{q \in x} v(q)\right]\right] & \left.=\lim _{\frac{1}{\lambda} \rightarrow 0}\left[\max _{p \in x}\left[u(p)+\frac{1}{\lambda} v(p)\right]-\frac{1}{\lambda} \max _{q \in x} v(q)\right]\right] \\
& \left.=\inf _{\lambda>0}\left[\max _{p \in x}\left[u(p)+\frac{1}{\lambda} v(p)\right]-\frac{1}{\lambda} \max _{q \in x} v(q)\right]\right]
\end{aligned}
$$

with $\hat{\lambda}=\frac{1}{\lambda}$ and the last equality is due to Eq. (2). Intuitively, the equivalence is due to the "cost" formulation of the GP model. The lower value of GP coincides with the intensity of temptation going to infinity.

\section{Generalized healthy perfectionism}

To account for more general models we introduce the Generalized Healthy Perfectionism representation $V_{H P}$. It evaluates a menu according to:

$$
V_{H P}(x)=\max _{p \in x}[u(p)+v(p)]-\max _{q \in x} v(q)+\theta(x)
$$

where $u, v: \Delta(Z) \rightarrow \mathbb{R}$ are expected utility functions, $\theta: \mathcal{X} \rightarrow \mathbb{R}_{+}$with $\theta(x) \geq \theta(y)$ if $y \subseteq x$. The interpretation of $u$ and $v$ is the same of the perfectionism representation. The only difference is in the function $\theta: \mathcal{X} \rightarrow \mathbb{R}_{+}$. Monotonicity with respect to set inclusion implies a premium for flexibility, although such flexibility is not used in the second-period. ${ }^{7}$ An example of $\theta(x)$ is the non-linear perfectionism $\theta(x)=$ $f\left(\max _{p \in x} u(x)\right)$, for some monotone $f: \mathbb{R} \rightarrow \mathbb{R}_{+}$. The case of $\theta(x)=k \max _{p \in x} u(p)$ intersects the model of Kopylov (2012) only if $k \max _{p \in x} u(p) \geq 0$ for all $x \in \mathcal{X}$. Differently from the perfectionist representation, the additional value of larger menus is not necessarily due to the inclusion of normatively optimal options. For example,

\footnotetext{
${ }^{7}$ The second-period choice rule associated with the HP representation, given "sophistication", is $C(x)=$ $\operatorname{argmax}_{p \in x}[u(p)+v(p)]$, the same rule of GP and the perfectionist model of Kopylov (2012). Then, including additional options in the menu may increase the first-period value but it does not affect the second-period choice.
} 
consider $\theta(x)=l(x)$ where $l$ is the Lebesgue measure. Larger menus are more valuable regardless of their normative content.

A generalized healthy perfectionism representation may violate the independence axiom, ${ }^{8}$ unless the function $\theta$ is linear with respect to mixtures of menus, i.e. $\theta(\alpha x+(1-$ $\alpha) y)=\alpha \theta(x)+(1-\alpha) \theta(y)$. It may also violate the Perfection Set-Betweenness axiom of Kopylov (2012). Indeed, consider $p, q \in \Delta(Z)$ with $u(p)>u(q)$ and $v(q)>v(p)$ and $u(p)-(v(q)-v(p)) \geq u(q)$. The lottery $p$ is perfect in the menu $\{p, q\}$, hence Perfection Set Betweenness requires $p \succcurlyeq\{p, q\}$. However, assume $0=\theta(p)<\theta(\{p, q\})$, then $V_{H P}(p)=u(p)<\max _{p, q} u(p)-(v(q)-v(p))+\theta(\{p, q\})=V_{H P}(\{p, q\})$ Hence, $\{p, q\}$ is preferred to $p$ although $p$ is "perfect" in $\{p, q\}$. The previous preference is consistent with a "challenge against oneself" explanation. The individual benefits from the inclusion of a tempting option $q$ in the menu, since she may enjoy the challenge of resisting temptation.

The following theorem is the main result of the paper:

Theorem 2. For all $x \in \mathcal{X}$,

$$
\left.\inf _{\lambda>0}\left[\max _{p \in x}\left[u(p)+\frac{1}{\lambda} v(p)\right]-\frac{1}{\lambda} \max _{q \in x} v(q)\right]+\frac{1}{\lambda} \theta(x)\right]=\max _{p \in B(x ; v, \theta(x))} u(p)
$$

As for the perfectionism representation, the lower bound of the value assigned to a menu by the generalized healthy perfectionism is given by the willpower representation. In addition, the stock of willpower is identical to the perfectionism value and again perfectionism becomes willpower. The result can be used to better understand the axiomatic foundation of the willpower model. Since HP may violate independence and perfectionist set-betweenness, the same holds for the willpower representation. A particularly interesting case of Theorem 2 concerns the relation between the Limited Willpower model $w(x)=w$ for all $x \in \mathcal{X}$ and perfectionism. Let $\theta(x)=w$ for all $x \in \mathcal{X}$, then the HP representation is a "perturbation" of the GP representation:

$$
V_{H P}(x)=\max _{p \in x}\left[u(p)-\left(\max _{q \in x} v(q)-v(p)-w\right)\right]
$$

where the "perfectionism" value $w$ decreases the cost of resisting temptation by a fixed

\footnotetext{
${ }^{8}$ For all $x, y, z \in \mathcal{X}$ and $\alpha \in[0,1], x \succcurlyeq y$ implies $\alpha x+(1-\alpha) z \succcurlyeq \alpha y+(1-\alpha) z$.
} 
amount $w \geq 0$. In this case:

$$
\left.\inf _{\lambda>0}\left[\max _{p \in x}\left[u(p)+\frac{1}{\lambda} v(p)\right]-\frac{1}{\lambda} \max _{q \in x} v(q)\right]+\frac{1}{\lambda} w\right]=\max _{p \in B(x ; v, w)} u(p)
$$

where $B(x ; v, w)=\left\{p \in x: w \geq \max _{q \in x} v(q)-v(p)\right\}$. Limited willpower arises from a very specific case of healthy perfectionism. This result offers an indirect proof that the model of limited willpower satisfies the Independence and the Set Between axiom of Gul and Pesendorfer (2001).

In general, the utility of the willpower representation is a lower bound for the generalized healthy perfectionism, when the perfectionism value and the stock of willpower coincide (by point a. in Proposition 2):

$$
\max _{p \in x}[u(p)+v(p)]-\max _{q \in x} v(q)+\theta(x) \geq \max _{p \in B(x ; v, \theta(x))} u(p)
$$

The inequality is preserved if we let, as in Gul and Pesendorfer (2001), the intensity of the temptation and perfectionism to go to infinity.

Corollary 2. For all $x \in \mathcal{X}$,

$$
\lim _{\lambda \rightarrow \infty}\left[\max _{p \in x}[u(p)+\lambda v(p)]-\lambda \max _{q \in x} v(q)+\lambda \theta(x)\right] \geq \max _{p \in B(x ; v, \theta(x))} u(p)
$$

Differently from the temptation and self-control case $\theta(x)=0$ for all $x \in \mathcal{X}$, the previous inequality can be strict. Hence, the value of a menu for an overwhelmed perfectionist can be strictly larger than the value of the same menu for a willpower representation (with perfectionism equal to willpower).

\section{Uncertain temptation}

Uncertainty concerning the temptation that will strike in the second period is descriptively advantageous and technically useful (see Dekel and Lipman, 2012, for a discussion). The Strotz model is not continuous and small variations in the commitment utility $u$ may drastically change preferences. Uncertainty resolves this issue and it is often assumed in applications. Let $\mathcal{U}$ a subset of $\mathbb{R}^{n}$ and $\mu$ a probability measure on $\mathcal{U}$ endowed with the Borel $\sigma$-algebra. The Random GP with Healthy Perfectionism 
(RP) model is given by the following:

$$
V_{R P}(x)=\int_{\mathcal{U}}\left[\max _{p \in x}[u(p)+v(p)]-\max _{q \in x} v(q)+\theta(x)\right] \mu(d v)
$$

with $\theta(x) \geq \theta(y)$ if $y \subseteq x$. The individual is uncertain about the "type" of temptation that she will face, whereas the perfectionism value is certain. This assumption is plausible given the interpretation of perfectionism as a force that affects the first-period value. Since uncertainty concerns the second period choice, it is natural to assume a deterministic value for perfectionism. If $\theta(x)=0$ for all $x \in \mathcal{X}$, the RP model becomes the Random GP model of Dekel and Lipman (2012). A similar extension that accounts for uncertainty of future temptations in the Willpower model gives the Random Strotz with Willpower (RS) representation:

$$
V_{R S W}(x)=\int_{\mathcal{U}} \max _{p \in B(x ; v, w(x))} u(p) \mu(d v)
$$

where

$$
B(x ; v, w(x))=\left\{p \in x: w(x) \geq \max _{q \in x} v(q)-v(p)\right\}
$$

When $w(x)=0$ for all $x \in \mathcal{X}$, the model corresponds to the random Strotz model of Dekel and Lipman (2012). The next theorem is a generalization of Theorem 2:

Theorem 3. For all $x \in \mathcal{X}$,

$$
\int_{\mathcal{U}} \inf _{\lambda>0}\left[\max _{p \in x}\left[u(p)+\frac{1}{\lambda} v(p)\right]-\frac{1}{\lambda} \max _{q \in x} v(q)+\frac{1}{\lambda} \theta(x)\right] \mu(d v)=\int_{\mathcal{U}} \max _{p \in B(x ; v, \theta(x))} u(p) \mu(d v)
$$

The interpretation of the expression on the left-hand side is the following: it is the expected lower bound for the evaluation of a menu as the temptation and perfectionism intensities are free to vary. A special case of the previous result implies that the limit of the random GP model is given by the random Strotz model.

Corollary 3. If $\theta(x)=0$ for all $x \in \mathcal{X}$, i.e. no-perfectionism,

$$
\lim _{\lambda \rightarrow \infty} \int_{\mathcal{U}}\left[\max _{p \in x}[u(p)+\lambda v(p)]-\lambda \max _{q \in x} v(q)\right] \mu(d v)=\int_{\mathcal{U}} \max _{x \in B(x ; v, 0)} u(p) \mu(d v)
$$

where $B(x ; v, 0)=\left\{p \in x: v(p) \geq \max _{q \in x} v(q)\right\}$. 


\subsection{Perfectionism and random willpower}

In the last section we prove that, under uncertainty, the relation between perfectionism and willpower goes beyond the results above. Indeed, we will extend the result of Dekel and Lipman (2012) to random perfectionism and willpower. They showed that a preference has a sufficiently smooth random Strotz representation, if and only if, it has a random GP representation. We prove that the result can be generalized to account for willpower and perfectionism, when a condition on the perfectionism representation is met. A preference $\succcurlyeq$ has a sufficiently smooth RS with willpower representation, if and only if, it has a capped ${ }^{9} \mathrm{RGP}$ with perfectionism representation. To illustrate one direction of the result consider

$$
\int_{0}^{1} \max _{p \in B(x ; v+t u, w(x))} u(p) d t
$$

it is a RS with willpower $w(x)$ and a uniform intensity of temptation $v+t u$ and $t \sim U[0,1]$. Smaller values of $t$ correspond to a stronger intensity of the temptation. For each $t$, the set $B(x ; v+t u, w(x))$ is larger than the corresponding set of the random Strotz representation, $B(x ; v+t u, 0)$. Therefore,

$$
\max _{p \in B(x ; v+t u, w(x))} u(p) \geq \max _{p \in B(x ; v+t u, 0)} u(p)
$$

and for each $t \in[0,1]$ there exists a positive number $\theta_{t}(x)$ such that, $\max _{p \in B(x ; v+t u, w(x))} u(p)=$ $\max _{p \in B(x ; v+t u, 0)} u(p)+\theta_{t}(x)$. Each willpower representation assigns a higher value to a menu than its related Strotz representation. When aggregating over the different intensities of the temptation we have:

$$
\int_{0}^{1} \max _{p \in B(x ; v+t u, w(x))} u(p) d t=\int_{0}^{1} \max _{p \in B(x ; v+t u, 0)} u(p) d t+\theta(x)
$$

where $\theta(x)=\int_{0}^{1} \theta_{t}(x) d t$. By Dekel and Lipman (2012), the integral on the right-hand side of Eq. (1) is equal to a GP representation, hence

$$
\int_{0}^{1} \max _{p \in B(x ; v+t u, w(x))} u(p) d t=\max _{p \in x}[u(p)+v(p)]-\max _{q \in x} v(q)+\theta(x)
$$

\footnotetext{
${ }^{9}$ See the definition before Theorem 4 .
} 
a (random) GP with perfectionism representation. Adding uncertainty on the temptation $v$ gives

$\int_{\mathcal{U}}\left(\int_{0}^{1} \max _{p \in B(x ; v+t u, w(x))} u(p) d t\right) \mu(d v)=\int_{\mathcal{U}}\left(\max _{p \in x}[u(p)+v(p)]-\max _{q \in x} v(q)+\theta(x)\right) \mu(d v)$

To generalize the previous argument, we define a Continuous Intensity RS with Willpower the following representation:

$$
V_{C I}(x)=\int_{\mathcal{U}}\left(\int_{0}^{1} \max _{p \in B(x ; v+t u, w(x))} u(p) f(t \mid v) d t\right) \mu(d v)
$$

where $f(t \mid v)$ is a lower-semicontinuous density ${ }^{10}$ (see Dekel and Lipman, 2012, for details). We have the following result:

Proposition 1. Every Continuous Intensity RS with willpower has a RGP with perfectionism representation.

Differently from the random Strotz case, it is not true that any RGP with perfectionism has a RS with willpower representation. For example, consider the healthy perfectionist value of a menu $x=\{p, q\}$ with $v(p)=v(q)=m$, then $V_{H P}(x)=$ $\max _{p \in x} u(p)+\theta(x)$. Let $u(p)>u(q)$ then, if $\theta(x)>0$ and $\theta(p)=0, x \succ_{P} p$, since $V_{H P}(x)=u(p)+\theta(x)>u(p)=V_{H P}(p)$. Now consider a RS with willpower, $V_{R S W}=$ $\int_{\mathcal{U}} \max _{p \in B(x ; v, w(x))} \bar{u}(p) \mu(d v)$, with $\bar{u}(p)>\bar{u}(q)$ and $v(p)=v(q)=m$. For any stock of willpower $w(x) \geq 0, V_{R S W}(p)=\bar{u}(p)=\max _{p \in x} \bar{u}(p)=\int_{\mathcal{U}} \max _{p \in B(x ; v, w(x))} \bar{u}(p) \mu(d v)=$ $V_{R S W}(x)$. Then, $p \sim_{R S W} x$, which is inconsistent with the perfectionist ranking. Perfectionism strive can be strong enough to generate a strict preference for flexibility, whereas willpower cannot.

The next theorem shows that the previous example captures the unique case in which the two representations can differ. We say that a RGP with perfectionism $V_{R P}$ is capped if

$$
\max _{p \in x} u(p) \geq \int_{\mathcal{U}}\left(\max _{p \in x}[u(p)+v(p)]-\max _{q \in x} v(q)+\theta(x)\right) \mu(d v) \quad \forall x \in \mathcal{X}
$$

The value assigned to a menu by a capped "perfectionism" is bounded by a pure preference for flexibility.

\footnotetext{
${ }^{10} \mathrm{~A}$ density $f(t \mid v)$ is lower-semicontinuous if the sets $\{t: f(t)>k\}$ are open for all $k$.
} 
Theorem 4. A preference $\succcurlyeq$ has capped $R G P$ with perfectionism representation, if and only if, it has a Continuous Intensity $R S$ with willpower representation.

Theorem 4 closes the gap between random willpower and random perfectionism showing that the two are essentially equivalent when perfectionism is "bounded" by a pure preference for flexibility. The previous result can be useful to study the axiomatic foundations of the two models. As in the case of Dekel and Lipman (2012), the two models are observationally distinguishable if one can observe both choices among and from menus.

\section{An application to the dynamic of willpower and perfectionism}

In this last section we consider a dynamic application of the RS with willpower model, in the recursive setting of Gul and Pesendorfer (2007). We define inductively $Z_{1}=\mathcal{K}(\Delta(C))$ and for $t>1, Z_{t}=\mathcal{K}\left(\Delta\left(C \times Z_{t-1}\right)\right)$, where $\mathcal{K}(M)$ is the set of compact subsets of a set $M$ and $C$ is the set of consumption. Standard results imply the existence of a compact metric $Z_{\infty}$ which is homeomorphic to $\mathcal{K}\left(\Delta\left(C \times Z_{\infty}\right)\right)$ (see Gul and Pesendorfer, 2007, for details), with $Z_{\infty}$ representing infinite-horizon menus. Preferences $\succcurlyeq$ are defined on $\Delta\left(C \times Z_{\infty}\right)$ and a lottery $p \in \Delta\left(C \times Z_{\infty}\right)$ gives consumption $c$ in the current period and a continuation menu $z \in Z_{\infty}$. We assume that elements in the consumption set $C$ are represented by a pair $(c, d)$, where $d$ is a tempting good and $c$ is not. Lastly, we allow preferences to depend on a state $s \in S$, hence we have a family $\left\{\succcurlyeq_{s}\right\}_{s \in S}$. Let consider a recursive RS with willpower

$$
V(z, s)=\int_{0}^{1} \max _{(c, d, x) \in B(z, v+l u, w(s))} U(c, d, x, s) d l
$$

where $U(c, d, x, s)=u(c, d)+\delta V\left(x, s^{\prime}\right)$. We assumed that the only parameter depending on the state $s$ is the stock of willpower. For example, we can assume the existence of a function $\sigma: S \rightarrow \mathbb{R}_{+}$with $w(s)=\sigma(s) w$ and $w \geq 0$. For example, let $s=\max _{(c, d, x) \in z} v(d)-v\left(d^{*}\right)$, where $d^{*}$ is the yesterday's consumption of the tempting good. If $\sigma(s)$ is decreasing, resisting temptation yesterday decreases the amount of willpower today, modeling willpower as a limited resource. With less willpower, the ability to resist temptation is lower, leading to higher consumption of the tempting 
good $d$ today. If the function $\sigma(s)$ goes to 0 as $s$ increases, the individual enters a steady state where $\sigma(s) w=0$ and she is "trapped" in a (random) self-indulgent behavior. The nature of the steady-state is different from the overwhelming temptation, since temptations' intensity is constant over time. The random self-indulgent steadystate follows from the stock of willpower being used up. Decreasing willpower, also diminishes the value of perfectionism in the associated representation (By Proposition 1 there is one):

$$
V(z, s)=\max _{(c, d, x, s) \in z}\left[u(c, d)+v(d)+\delta V\left(x, s^{\prime}\right)\right]-\max _{(c, d, x, s) \in z} v(d)+\theta(s, z)
$$

resisting temptation yesterday warns the individual of the drawbacks of larger menus. If willpower depletes completely, $\theta(s, z)=0$ and the value of perfectionism disappears.

In the case of an increasing function $\sigma(s)$, we model how individuals build willpower. Resisting temptation yesterday increases the stock of willpower today, as predicted by the Strength Model of Self-control (Baumeister et al., 1998). Willpower behaves as a muscle, it can be "trained" and become stronger. Again, a steady-state can be reached if the stock of willpower is big enough, in this case the individual has a pure preference for flexibility:

$$
V(z, s)=\max _{(c, d, x) \in z} U(c, d, x, s)
$$

At the same time, increasing willpower over time increases the value of perfectionism $\theta(s, z)$ in the associated representation. Resisting temptation produces a form of "perfectionism" addiction, the individual assigns an ever greater value to perfectionism (actually flexibility), ending in a pure preference for flexibility.

\section{Conclusion}

The Strotz model corresponds to a limiting case of the Gul and Pesendorfer (2001) model in which temptation becomes overwhelming. This result is a particular case of a more general relation between perfectionism and willpower. When temptation and perfectionism vary, the lower bound for the value of a menu is given by the value attached to the menu by a model of willpower. Moreover, the stock of willpower is exactly equal to the value of perfectionism. The result is preserved when temptation 
is uncertain. In addition, we extend the link between perfectionism and willpower providing conditions for the equivalence of the RS with willpower representation and the RGP with perfectionism representation. 


\section{Appendix A: $\epsilon$-subdifferentials}

Let $X$ be a locally convex vector space and $f: X \rightarrow \mathbb{R}$ a convex function. Let $\operatorname{dom} f=\{x \in X: f(x)<+\infty\}$. $f$ is proper if $\operatorname{dom} f \neq \emptyset$ and $f>-\infty$ for all $x$. The $\epsilon$-directional derivative of $f$ at $v$ in the direction $u$ is given for a fixed $\epsilon \geq 0$ by (see Zalinescu (2002))

$$
f_{\epsilon}^{\prime}(v, u) \triangleq \inf _{t>0} \frac{f(v+t u)-f(v)+\epsilon}{t}
$$

The $\epsilon$-subdifferential of $f$ at $v \in X, \partial_{\epsilon} f: X \rightrightarrows X^{*}$, is defined as

$$
\partial_{\epsilon} f(v)=\left\{x^{*} \in X^{*}:\left\langle u-v, x^{*}\right\rangle \leq f(v)-f(u)+\epsilon\right\}
$$

If $f$ is proper and convex, the following properties hold for every $\epsilon \geq 0$ :

Proposition 2 (Zalinescu (2002)).

(a). $f_{\epsilon}^{\prime}(v, u) \leq f(v+u)-f(v)+\epsilon$, for all $u \in X$.

(b). $f_{\epsilon}^{\prime}(v, u)=\max _{x^{*} \in \partial_{\epsilon} f(v)}\left\langle u, x^{*}\right\rangle$.

(c.) If $f$ is sublinear, $\partial_{\epsilon} f(v)=\left\{x^{*} \in \partial f(0):\left\langle v, x^{*}\right\rangle \geq f(v)-\epsilon\right\}$, where $\partial f=\partial_{0} f$ is the standard subdifferential.

(d). $0 \leq \epsilon_{1} \leq \epsilon_{2}<\infty$ implies

$$
f_{0}^{\prime}(v, u) \leq f_{\epsilon_{1}}^{\prime}(v, u) \leq f_{\epsilon_{2}}^{\prime}(v, u)
$$

If $f: X \rightarrow \mathbb{R}$ is proper and convex and $v \in \operatorname{dom} f$. Then for every $u \in X$

$$
f_{0}^{\prime}(v ; u)=\lim _{t \rightarrow 0} \frac{f(v+t u)-f(v)}{t}=\inf _{t>0} \frac{f(v+t u)-f(u)}{t}
$$

\section{Appendix B: Proofs}

Proof. Of Theorem 2. For a fixed menu $x \in \mathcal{X}$, consider $V_{x}(v)=\max _{p \in x} v(p)$, as a function $V_{x}(v): \mathbb{R}^{n} \rightarrow \mathbb{R}$. The expression $\max _{p \in x}\left[u(p)-\lambda\left(\max _{q \in x} v(q)-v(p)\right)\right]+\lambda \theta(x)$ can be rewritten as

$$
\frac{\left.\max _{p \in x}\left[v(p)+\frac{1}{\lambda} u(p)\right]-\max _{q \in x} v(q)\right]+\theta(x)}{1 / \lambda}
$$

Defining $\epsilon=\theta(x)$ and $\hat{\lambda}=1 / \lambda$, the left-hand side of the equation in the statement becomes

$$
\inf _{\hat{\lambda}>0} \frac{\left.\max _{p \in x}[v(p)+\hat{\lambda} u(p)]-\max _{q \in x} v(q)\right]+\epsilon}{\hat{\lambda}}
$$

The previous expression is the $\epsilon$-subderivative of the function $V_{x}(v)=\max _{p \in x} v(q)$ in the direction $u \in \mathbb{R}^{n}$ (see Appendix A) and we denoted it by $V_{x}^{\prime}(v, u)$. By Property (b). in Proposition 2,

$$
V_{x}^{\prime}(v, u)=\max _{k \in \partial_{\epsilon} V_{x}(v)} u(k)
$$

By point $(c)$. in Prop. 2, $\partial_{\epsilon} V_{x}(v)=\left\{k \in \partial V_{x}\left(0_{n}\right): v(k) \geq V_{x}(v)-\epsilon\right\}$, but $\partial V_{x}\left(0_{n}\right)=$ $\overline{\mathrm{co}} x=x$, since $x \in \mathcal{X}$ is compact and convex. Therefore, $\partial_{\epsilon} V_{x}(v)=\left\{p \in x: v(p) \geq V_{x}(v)-\epsilon\right\}$ and $V_{x}^{\prime}(v, u)=\max _{p \in B(x, v, \epsilon)} u(p)$, as desired. 
Proof. Of Corollary 2. The result follows from

$$
\begin{aligned}
& \lim _{\lambda \rightarrow \infty}\left[\max _{p \in x}\left[u(p)-\lambda\left(\max _{q \in x} v(q)-v(p)\right)\right]+\lambda \theta(x)\right] \\
= & \lim _{\lambda \rightarrow \infty} \lambda\left[\max _{p \in x}\left[\lambda^{-1} u(p)-\left(\max _{q \in x} v(y)-v(q)\right)\right]+\theta(x)\right] \\
= & \left.\lim _{\hat{\lambda} \rightarrow 0}\left[\frac{1}{\hat{\lambda}}\left(\max _{p \in x}[v(p)+\hat{\lambda} u(p)]-\max _{q \in x} v(q)\right]+\theta(x)\right)\right]
\end{aligned}
$$

where in the last equality $\hat{\lambda}=\lambda^{-1}$. To conclude,

$$
\begin{aligned}
& \left.\lim _{\hat{\lambda} \rightarrow 0}\left[\frac{1}{\hat{\lambda}}\left(\max _{p \in x}[v(p)+\hat{\lambda} u(p)]-\max _{q \in x} v(q)\right]+\theta(x)\right)\right] \\
\geq & \left.\inf _{\hat{\lambda}>0}\left[\frac{1}{\hat{\lambda}}\left(\max _{p \in x}[v(p)+\hat{\lambda} u(p)]-\max _{q \in x} v(q)\right]+\theta(x)\right)\right] \\
= & \max _{p \in B(x ; v, \theta(x))} u(p)
\end{aligned}
$$

where the last equality follows from Theorem 2 .

Proof. Of Corollary 3.

$$
\begin{aligned}
& \lim _{\lambda \rightarrow \infty} \int_{\mathcal{U}}\left[\max _{p \in x}\left[u(p)-\lambda\left(\max _{q \in x} v(q)-v(p)\right)\right]\right] \mu(d v) \\
= & \lim _{\lambda \rightarrow \infty} \lambda\left(\int_{\mathcal{U}} \max _{p \in x} v(p)+\lambda^{-1} u(p)-\max _{q \in x} v(q) d \mu(d v)\right) \\
= & \lim _{\hat{\lambda} \rightarrow 0} \int_{\mathcal{U}} \frac{\max _{p \in x} v(p)+\hat{\lambda} u(p)-\max _{q \in x} v(q)}{\hat{\lambda}} \mu(d v) \\
= & \int_{\mathcal{U}} \lim _{\hat{\lambda} \rightarrow 0} \frac{\max _{p \in x} v(p)+\hat{\lambda} u(p)-\max _{q \in x} v(q)}{\hat{\lambda}} \mu(d v) \\
= & \int_{\mathcal{U}} \inf _{\hat{\lambda}>0} \frac{\max _{p \in x} v(p)+\hat{\lambda} u(p)-\max _{q \in x} v(q)}{\hat{\lambda}} \mu(d v) \\
= & \int_{\mathcal{U}} \max _{p \in B(x ; v, 0)} u(p) d \mu(v)
\end{aligned}
$$

where the third equality follows from the Dominated Convergence theorem, the forth equality from convexity of the function $\max _{p \in x} v(q)$ and Eq. (2) and the last equality from Theorem 3.

To prove Proposition 1, we need the following result:

Proposition 3. For all $w(x) \geq 0$, there exists $\theta(x) \geq 0$, such that

$$
\int_{\mathcal{U}} \max _{p \in B(x ; v, w(x))} u(p) \mu(d v)=\int_{\mathcal{U}} \max _{p \in B(x ; v, 0)} u(p) \mu(d v)+\theta(x)
$$

Proof. Of Proposition 3. By point (d). of Proposition 2, for all $x \in \mathcal{X}, w(x) \geq 0$ implies

$$
\max _{p \in B(x ; v, w(x))} u(p) \geq \max _{p \in B(x ; v, 0)} u(p)
$$

Then, for each $v \in \mathcal{V}$, there exists $\theta_{v}(x) \geq 0$ such that

$$
\max _{p \in B(x ; v, w(x))} u(p)=\max _{p \in B(x ; v, 0)} u(p)+\theta_{v}(x)
$$


Integrating over $\mathcal{U}$,

$$
\int_{\mathcal{U}} \max _{p \in B(x ; v, 0)} u(p) \mu(d v)+\theta(x)=\int_{\mathcal{U}} \max _{p \in B(x ; v, w(x))} u(p) \mu(d v)
$$

where $\theta(x)=\int_{\mathcal{U}} \theta_{v}(x) \mu(d v)$.

Proof. Of Proposition 1. For each $x \in \mathcal{X}$

$$
\begin{aligned}
& \int_{\mathcal{U}}\left(\int_{0}^{1} \max _{p \in B(x ; v+t u, w(x))} u(p) f(t \mid v) d t\right) \mu(d v) \\
= & \int_{\mathcal{U}}\left(\int_{0}^{1} \max _{p \in B(x ; v+t u, 0)} u(p) f(t \mid v) d t+\theta(x)\right) \mu(d v) \\
= & \int_{\mathcal{U}} \max _{p \in x}[u(p)+v(p)]-\max _{q \in x} v(q)+\theta(x) \mu(d v)
\end{aligned}
$$

Where the first equality follows from Proposition 3 and the second from Dekel and Lipman (2012, Th. 2).

Proof. Of Theorem 4. By Dekel and Lipman (2012),

$$
\int_{\mathcal{U}} \max _{p \in x}[u(p)+v(p)]-\max _{q \in x} v(q) \mu(d v)+\theta(x)=\int_{\mathcal{U}} \int_{0}^{1} \max _{p \in B(x ; v+t u, 0)} u(p) d t \mu(d v)+\theta(x)
$$

Then, we have to find $w(x) \geq 0$ such that

$$
\theta(x)=\int_{\mathcal{U}}\left(\int_{0}^{1} \max _{p \in B(x ; v+t u, w(x))} u(p) d t-\int_{0}^{1} \max _{p \in B(x ; v+t u, 0)} u(p) d t\right) \mu(d v)
$$

The range of the right-hand side of the previous equation as $w(x)$ varies is $[0, k]$, where 0 occurs for $w(x)=0$ and $k=\max _{p \in x} u(p)-\left(\int_{\mathcal{U}} \max _{p \in x}[u(p)+v(p)]-\max _{q \in x} v(q) \mu(d v)\right)$. Since $V_{R P}$ is capped, $\theta(x) \in[0, k]$. For each $w(x) \triangleq \bar{w}$ define $\succcurlyeq_{\bar{w}}$ in the following way: $x \succcurlyeq_{\bar{w}} y$, if and only if,

$$
\int_{\mathcal{U}} \int_{0}^{1} \max _{p \in B(x ; v+t u, \bar{w})} u(p) d t \mu(d v) \geq \int_{\mathcal{U}} \int_{0}^{1} \max _{p \in B(y ; v+t u, \bar{w})} u(p) d t \mu(d v)
$$

clearly, all $\succcurlyeq_{\bar{w}}$ agree on singletons. Let $p(\bar{w}) \in x$ such that

$$
u(p(\bar{w}))=\int_{\mathcal{U}} \int_{0}^{1} \max _{p \in B(x ; v+t u, \bar{w})} u(p) d t \mu(d v)
$$

then $u(p(\bar{w})) \geq u\left(p\left(\bar{w}^{\prime}\right)\right)$ if $\bar{w} \geq \bar{w}^{\prime}$ and $\bar{w} \mapsto u(p(\bar{w}))$ is monotone. By the cap condition $\theta(x) \in[0, u(p(\hat{w}))-u(p(0))]$, for $\hat{w}(x)$ great enough. Suppose that $\lim _{\bar{w}_{n} \rightarrow \bar{w}^{+}} u\left(p\left(\bar{w}_{n}\right)\right)>$ $\lim _{\bar{w}_{n} \rightarrow \bar{w}^{-}} u\left(p\left(\bar{w}_{n}\right)\right)$ and consider $\succcurlyeq_{w^{\prime}} \triangleq \succcurlyeq$ on singletons (the coincides for all $\left.w^{\prime}\right)$. This implies either $p\left(\bar{w}_{n}\right) \succcurlyeq q$ and $q \succ p(\bar{w})$ or $q \succcurlyeq p\left(\bar{w}_{n}\right)$ and $p(\bar{w}) \succ q$, for some $q \in \Delta(Z)$, both contradict continuity. Then there exists $w^{*}$ such that $\theta(x)=u\left(p\left(w^{*}\right)-u(p(0))\right.$. By the definition of $p\left(w^{*}\right)$,

$$
\begin{aligned}
\int_{\mathcal{U}} \int_{0}^{1} \max _{p \in B\left(x ; v+t u, w^{*}\right)} u(p) d t \mu(d v) & =\int_{\mathcal{U}} \int_{0}^{1} \max _{p \in B(x ; v+t u, 0)} u(p) d t \mu(d v)+\theta(x) \\
& =\int_{\mathcal{U}} \max _{p \in x}[u(p)+v(p)]-\max _{q \in x} v(q) \mu(d v)+\theta(x)
\end{aligned}
$$

as desired. To see the opposite, notice that the proof of Proposition 1 implies that the RGP with perfectionism associated with the RS with willpower is capped. 


\section{References}

Ahn, D. S. (2008). Ambiguity without a state space. The Review of Economic Studies $75(1), 3-28$.

Aleskerov, F., D. Bouyssou, and B. Monjardet (2007). Utility maximization, choice and preference, Volume 16. Springer Science \& Business Media.

Baumeister, R. F., E. Bratslavsky, M. Muraven, and D. M. Tice (1998). Ego depletion: is the active self a limited resource? Journal of personality and social psychology $74(5), 1252$.

De Oliveira, H., T. Denti, M. Mihm, and M. K. Ozbek (2013). Rationally inattentive preferences. Available at SSRN 2274286.

Dekel, E. and B. L. Lipman (2012). Costly Self Control and Random Self Indulgence. Econometrica 80(3), 1271-1302.

DellaVigna, S. and U. Malmendier (2006). Paying not to go to the gym. American Economic Review 96(3), 694-719.

Ergin, H. and T. Sarver (2010). A unique costly contemplation representation. Econometrica 78(4), 1285-1339.

Frick, M. (2015). Monotone threshold representations. Theoretical Economics, Forthcoming.

Frost, R. O., P. Marten, C. Lahart, and R. Rosenblate (1990). The dimensions of perfectionism. Cognitive therapy and research 14(5), 449-468.

Gajdos, T., T. Hayashi, J.-M. Tallon, and J.-C. Vergnaud (2008). Attitude toward imprecise information. Journal of Economic Theory 140(1), 27-65.

Gul, F. and W. Pesendorfer (2001). Temptation and self-control. Econometrica 69(6), $1403-1435$.

Gul, F. and W. Pesendorfer (2007). Harmful addiction. The Review of Economic Studies 74 (1), 147-172.

Hamachek, D. E. (1978). Psychodynamics of normal and neurotic perfectionism. Psychology: A Journal of Human Behavior.

Kopylov, I. (2012). Perfectionism and choice. Econometrica 80(5), 1819-1843.

Kreps, D. M. (1979). A representation theorem for" preference for flexibility". Econometrica: Journal of the Econometric Society, 565-577.

Manzini, P., M. Mariotti, and C. J. Tyson (2013). Two-stage threshold representations. Theoretical Economics 8(3), 875-882.

Masatlioglu, Y., D. Nakajima, and E. Ozdenoren (2011). Revealed willpower. Technical report, mimeo.

Noor, J. and N. Takeoka (2015). Menu-dependent self-control. Forthcoming: Journal of Mathematical Economics. 
Ozdenoren, E., S. W. Salant, and D. Silverman (2012). Willpower and the optimal control of visceral urges. Journal of the European Economic Association 10(2), $342-368$.

Pennesi, D. (2015). Costly information acquisition and the temporal resolution of uncertainty. Journal of Mathematical Economics 60, 115 - 122.

Stoeber, J. and K. Otto (2006). Positive conceptions of perfectionism: Approaches, evidence, challenges. Personality and social psychology review 10(4), 295-319.

Strotz, R. H. (1956). Myopia and inconsistency in dynamic utility maximization. The Review of Economic Studies 23(3), 165-180.

Zalinescu, C. (2002). Convex analysis in general vector spaces. World Scientific. 


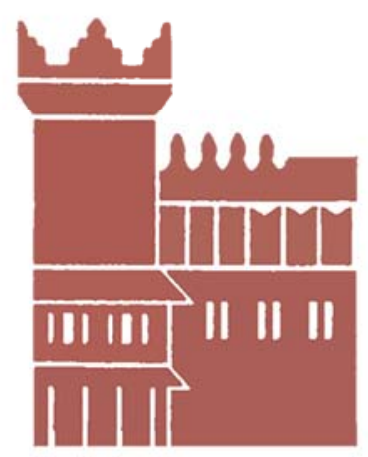

Alma Mater Studiorum - Università di Bologna DEPARTMENT OF ECONOMICS

Strada Maggiore 45

40125 Bologna - Italy

Tel. +39051 2092604

Fax +390512092664

http://www.dse.unibo.it 